\title{
Compatibilidade de Metarhizium anisopliae (Metschnikoff) Sorokin (Hypocreales: Clavicipitaceae) com Chrysoperla externa (Hagen) (Neuroptera: Chrysopidae)
}

\author{
Eunice Cláudia Schlick Souza ${ }^{\bowtie}$, Luciana Claudia Toscano², Genivaldo David de Souza Schlick³, \\ André Júnior Andrade Peres ${ }^{2}$, Pamella Mingotti Dias ${ }^{2}$ \& Wilson Itamar Maryama ${ }^{2}$
}

\begin{abstract}
1. Embrapa Agropecuária Oeste em Ponta Porã MS, e-mail: euniceschlick@hotmail.com (Autor para correspondência ${ }^{\bowtie}$ ). 2. Programa de Pós-graduação em Agronomia "Sustentabilidade na Agricultura", Universidade Estadual de Mato Grosso do Sul - Cassilândia -MS., e-mail: toscano@uems.br, ajaperes@hotmail.com, pamellamingotti@hotmail.com, maryama@uems.br. 3. Instituto Federal de Mato Grosso do Sul - Câmpus
\end{abstract} Ponta Porã - MS, e-mail: genivaldo.schlick@ifms.edu.br.

\section{EntomoBrasilis 8 (3): 189-195 (2015)}

Resumo. Os agroecossistemas contem vários agentes de controle biológico atuando simultaneamente sobre diferentes ou o mesmo inseto alvo. A cultura do algodoeiro apresenta um rico complexo de inimigos naturais associados às pragas, dentre eles, destaca-se o predador Chrysoperla externa (Hagen) e o fungo entomopatogênico Metarhizium anisopliae (Metschnikoff) Sorokin. O objetivo deste trabalho foi avaliar a compatibilidade do fungo M. anisopliae sobre os aspectos biológicos do predador C. externa. O primeiro ensaio avaliou o desenvolvimento de C. externa quando tratado com as seguintes suspensões de $M$. anisopliae: T1- água (testemunha); T2-10 ${ }^{5}$ conídios. $\mathrm{mL}^{-1}$ de $M$. anisopliae (M.a.); $\mathrm{T}_{3}-10^{6} \mathrm{conídios.mL}^{-1}$ de $M . a$.; T4- $10^{7}$ conídios.mL ${ }^{-1}$ de M.a.;T5- $10^{8}$ conídios. $\mathrm{mL}^{-1}$ de M.a. No segundo ensaio foram avaliadas a duração das fases larval e pupal do predador, bem como a mortalidade larval em cada tratamento. Para isto, ovos de C. externa foram individualizados em placas de Petri e mantidos em laboratório. Quando as larvas de C. externa eclodiram, foram oferecidas ninfas de mosca-branca em discos foliares oriundos do algodoeiro tratado com: T1 -água

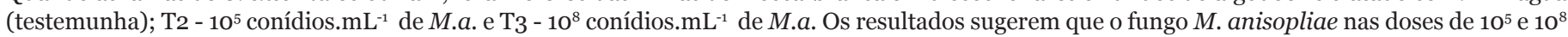
conídios.mL ${ }^{-1}$ de M.a. não interferem nos aspectos biológicos do predador C. externa.

Palavras-chave: Controle microbiano de insetos; Crisopídeo; Fungos entomopatogênicos; Mosca-branca.

\section{Compatibility Metarhizium anisopliae (Metschnikoff) Sorokin (Hypocreales: Clavicipitaceae) with Chrysoperla externa (Hagen) (Neuroptera: Chrysopidae)}

Abstract. The ecoagrossistemas several biological control agents acting simultane ously on different or the same target insect. The culture of cotton presents a rich complex of natural enemies associated with pests, among which stands out the predator Chrysoperla externa (Hagen) and the entomopathogenic fungus Metarhizium anisopliae (Metschnikoff) Sorokin. The objective of this study was to evaluate the compatibility of the fungus $M$. anisopliae on the biological aspects of the predator C. externa. The first study evaluated the development of $C$. externa when treated with the following suspensions of M. anisopliae: T1 water (control); T2- $10^{5}$ conídios.mL $\mathrm{mL}^{-1} \mathrm{de}$ M. anisopliae (M.a.); T3- 10 ${ }^{6}$ conídios.mL $^{-1}$ de $M . a$.; T4- 10 conídios.mL ${ }^{-1}$ de M.a.;T5- $10^{8}$ conídios.mL $\mathrm{mL}^{-1}$ de M.a.. The second assay measured the duration of the larval and pupal predator and larval mortality in each treatment. For this, C. externa eggs were individualized in Petri dishes and kept under laboratory conditions. When the larvae of $C$. externa

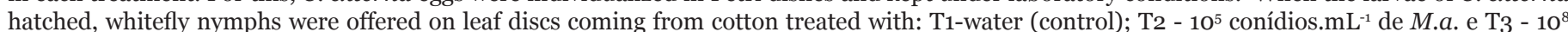

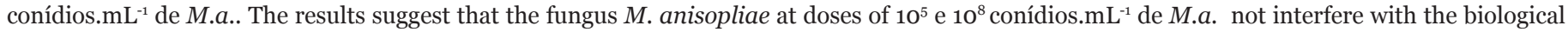
aspects of the predator C. externa.

Keywords: Entomopathogenic fungi; Green lacewing; Microbial control of insects; Whitefly.

30 entro do ecossistema agrícola, os agentes de controle biológico podem ocorrer simultaneamente atuando em diferentes ou o mesmo inseto alvo (França et al. 2006). Os fungos entomopatogênicos dependem de fatores abióticos e bióticos para o seu desenvolvimento e disseminação. Porém, os insetos predadores exploram grandes áreas foliares em busca de suas presas, estando suscetível ao entomopatógeno presente nas folhas, através de aplicação ou, ainda, pela ingestão de presas infectadas (FRANÇA et al. 2006). A cultura do algodoeiro Gossypium hirsutum L. apresenta um rico complexo de inimigos naturais associados, entre eles predadores como Chrysoperla externa (Hagen) (RAMiro \& FARIA 2006) e o fungo Metarhizium anisopliae (Metschnikoff) Sorokin (Gravena 2000).

Dentre o complexo de pragas que ataca a cultura do algodão, a mosca-branca Bemisia tabaci (Gennadius) biótipo B (Hemiptera:
Aleyrodidae) ocasiona grandes perdas na lavoura de algodão em diversos países (Butler Junior et al. 1991; VAn EMden \& HaRrington 2007). Ao sugar a seiva das plantas, a mosca-branca excreta uma substância açucarada denominada de "honeydew", dando condições favoráveis para o crescimento do fungo Capnodium spp., responsável pela formação da fumagina, na qual deprecia a qualidade da fibra (BERRY et al. 2004).

Com o intuito de reduzir o impacto dos inseticidas, o controle biológico tem se caracterizado como uma alternativa muito promissora na supressão de populações de pragas que causam danos econômicos em culturas agrícolas, com destaque para o uso de microrganismos e insetos predadores (BuENo et al. 2012). O fungo M. anisopliae é o mais estudado dentre os microorganismos entomopatogênicos pela variedade de hospedeiros que apresenta, e pela ocorrência enzootica e epizootica em insetos-pragas (Alves 


\section{\& LOPES 2008).}

Pesquisas utilizando $M$. anisopliae revelam que este agente é patogênico para o psilídeo da goiabeira Triozoida limbata (Enderlein) (Hemiptera: Psyllidae), psilídeo Diaphorina citri Kuwayama (Hemiptera: Psyllidae), ácaro-rajado Tetranychus urticae ( Koch) (Acari: Tetranychidae) e o cascudinho Alphitobius diaperinus (Panzer) (Coleoptera: Tenebrionidae), a moscabranca B. tabaci biótipo B (AzEvedo et al. 2005; GASSEN 2006; PADUlla 2007; Rohde et al. 2006; TAMAI 2002).

LOUREIRO et al. 2012, testando vários isolados de $M$. anisopliae no controle da cigarrinha-da-raíz da cana-de-açúcar Mahanarva fimbriolata (Stål) (Hemiptera: Cercopidae) em condições de campo, verificaram 30 após a pulverização que os isolados IBCB 408 e IBCB 425 apresentaram eficiência no controle de ninfas de 63 e $62 \%$, respectivamente, obtendo $100 \%$ de eficiência para os adultos.

Quanto aos predadores, Chrysoperla externa (Hagen) (Neuroptera: Chrysopidae) tem se mostrado muito eficiente no controle de insetos nocivos como a mosca-branca devido a sua alta capacidade de predação, voracidade, generalismo e facilidade de criação em laboratório (AuAd et al. 2001; Silva et al. 2004; AuAd et al. 2005; CARVALHO \& SOUZA 2009; BEZERRA et al. 2009).

No Brasil, a liberação de ovos e ou larvas de espécies de crisopídeos para o controle de pragas tem poucos estudos relacionados (Pinto \& PARRA 2002; BARBOSA et al. 2008; CARVALHO \& Souza 2009), porém Souza (2013) observou que a utilização de copos plásticos e colmeia de papelão são eficientes métodos na liberação de larvas de $2^{\circ}$ instar de $C$. externa para a redução da densidade populacional de Macrosiphum euphorbiae (Thomas) (Hemiptera: Aphididae) na cultura da roseira em ambiente protegido.

Na busca de uma agricultura sustentável e com maior custobenefício, é de grande importância os estudos voltados para o manejo dos artrópodes-pragas aliados ao uso de agentes de controle biológico, reduzindo os níveis de danos econômicos causados por estes organismos. Deste modo, o objetivo deste trabalho foi avaliar a compatibilidade do fungo $M$. anisopliae sobre os aspectos biológicos do predador C. externa.

\section{MATERIAL E MÉTODOS}

A pesquisa foi realizada na Universidade Estadual de Mato Grosso do Sul - UEMS, Unidade de Cassilândia, MS.

Multiplicação de $\boldsymbol{B}$. tabaci. A população inicial de moscabranca para a criação massal foi proveniente de coletas realizadas em hortas localizadas no município de Cassilândia - MS, com auxílio de aspirador bucal. Posteriormente, as moscas-brancas foram transferidas para gaiolas de armação de ferro de $3 \mathrm{~m} \times 3 \mathrm{~m}$ x $2 \mathrm{~m}$ com tela anti-afídeo. Foram utilizadas plantas de tomate, soja, brócolis e couve no interior das gaiolas para a alimentação e reprodução de $B$. tabaci.

Multiplicação de $C$. externa. A população inicial de $C$. externa foi adquirida junto à Agência Paulista de Tecnologia dos Agronegócios Polo Extremo Oeste em Andradina - SP. Posteriormente, adultos de $C$. externa foram coletados semanalmente, com auxílio de rede entomológica no campo da área experimental da UEMS e transferidos para o laboratório sob temperatura de $25 \pm 2{ }^{\circ} \mathrm{C}$, UR de $60 \pm 10 \%$ e fotofase de 12 horas.

Os neurópteros foram identificados conforme descrição realizada por Freitas (2003). Em seguida, foram mantidos em gaiolas de PVC, com $23 \mathrm{~cm}$ de altura e $10 \mathrm{~cm}$ de diâmetro, as quais foram revestidas internamente com papel sulfite branco como substrato para a oviposição. A extremidade superior das gaiolas foi vedada com tecido "voil" fixado com elástico e a extremidade inferior foram apoiadas em bandeja circular de PVC de 24 cm de diâmetro, forrado com papel toalha branco. Diariamente, foi fornecida uma dieta constituída de levedo de cerveja e mel, na proporção de 1:1. Como a dieta fica de consistência pastosa, ela foi pincelada a uma fita adesiva na parede do papel, enquanto a água deionizada foi fornecida através de chumaço de algodão (RIBEIRo 1988). Após 24 horas da oviposição, o papel contendo os ovos de C. externa era substituído, e os pedicelos dos ovos cortados com auxílio de uma tesoura. Os ovos foram individualizados em tubos de vidro de 2,5 cm de diâmetro por 8,5 cm de altura, vedados com filme de polietileno ou em placas de Petri de $9 \mathrm{~cm}$ de diâmetro, conforme a fase dos ensaios.

Experimento o1. Influência de $M$. anisopliae sobre o ciclo biológico do predador $\boldsymbol{C}$. externa. Para a análise das fases biológicas do predador C. externa, os indivíduos em seus respectivos estágios de desenvolvimento (ovos, larvas, pupas e adultos) foram obtidos da criação de manutenção. Utilizaramse tubos de ensaio de vidro de $2,5 \mathrm{~cm}$ de diâmetro x $8,5 \mathrm{~cm}$ de altura e placas de Petri de $9 \mathrm{~cm}$ de diâmetro, vedados com filme de polietileno, conforme a necessidade dos ensaios, para a contenção das diferentes fases do inseto.

Os ovos, os três instares larvais, pupas eadultos de C.externa foram pulverizados através de um pulverizador manual, utilizandose $100 \mathrm{~mL}^{-1}$ de suspensão de fungos entomopatogênicos, e posteriormente levados para o laboratório com temperatura de $25 \pm 2{ }^{\circ} \mathrm{C}$, umidade relativa de $60 \pm 10 \%$ e fotofase de 12 horas. As larvas foram alimentadas com ovos de Anagasta kuehniella (Zeller) (Lepidoptera: Pyralidae) durante toda a fase larval. As características biológicas avaliadas foram: duração do período de incubação (dias), porcentagem de larvas eclodidas (viabilidade), duração de cada instar (dias), porcentagem de mortalidade (\%), biomassa de dez larvas (do segundo e terceiro ínstares) a cada 24 horas após cada ecdise, porcentagens de emergência, ocorrência de deformações dos adultos emergidos, longevidade e a capacidade de oviposição.

Os tratamentos consistiram de diferentes doses do inseticida biológico Metarril $^{\circledR}$, cujo princípio ativo é o fungo $M$. anisopliae (isolado IBCB 425): T1- água (testemunha); T2-10 ${ }^{5}$ conídios. $\mathrm{mL}^{-1}$ de $M$. anisopliae (M.a.); $\mathrm{T}^{-}{ }^{-} 10^{6}$ conídios. $\mathrm{mL}^{-1}$ de M.a.; T4- $10^{7}$ conídios. $\mathrm{mL}^{-1}$ de M.a. e T5-10 ${ }^{8}$ conídios.mL ${ }^{-1}$ de M.a. O delineamento experimental foi o inteiramente casualizado, com cinco tratamentos (doses do fungo e testemunha), 20 repetições para ovos e 15 repetições para larvas, pupas e adultos de $C$. externa.

Experimento 02. Características biológicas de $C$. externa alimentada com ninfas de $B$. tabaci biótipo $B$ criadas em algodoeiro tratado com o fungo $M$. anisopliae. Ovos de $C$. externa provenientes da criação de manutenção foram individualizados em placas de Petri de $9 \mathrm{~cm}$ de diâmetro, vedadas na parte superior com filme de polietileno e mantidos a $25 \pm 2{ }^{\circ} \mathrm{C}$, UR $60 \pm 10 \%$ e fotofase de 12 horas.

Após a eclosão das larvas, foram oferecidas fases imaturas de mosca-branca em discos foliares de $5 \mathrm{~cm}$ de diâmetro oriundos de plantas de algodão tratadas ou não com $M$. anisopliae, após 24 horas da aplicação. Diariamente, os discos foram trocados mantendo-se o alimento em abundância para que não houvesse interferência na necessidade nutricional do predador para completar seu ciclo biológico.

As características biológicas avaliadas foram: a duração (dias) da fase larval e pupal e porcentagem de mortalidade (\%) de cada fase imatura. Os tratamentos foram constituídos por: T1 - água (testemunha); T2 - $10^{5}$ conídios. $\mathrm{mL}^{-1}$ de M.a. e T3 - $10^{8}$ conídios. $\mathrm{mL}^{-1}$ de $M . a$.

Utilizou-se o delineamento inteiramente casualizado, sendo a testemunha e duas doses do fungo uma mais prejudicial ao desenvolvimento da mosca-branca e a outra menos prejudicial 
ao predador (Schlick-Souza et al. 2011), com 20 repetições (larvas). Os dados obtidos em todos os ensaios foram submetidos ao teste de Kolmogorov-Smirnov para verificar se apresentavam distribuição normal. Quando isso ocorreu, foi utilizado o teste de Snedcor (F); já quando não apresentaram normalidade, os dados foram previamente transformados em $(x+0,5)^{1 / 2}$ e arco seno de $(\mathrm{x}+0,5)^{1 / 2}$ para normalizá-los. Quando existiu diferença entre os genótipos avaliados, foi realizado o teste de Tukey $(\mathrm{P}<0,05)$ para a comparação das médias.

\section{RESULTADOS E DISCUSSÃO}

Influência de $M$. anisopliae sobre o ciclo biológico do predador C. externa. Não ocorreram diferenças significativas entre os tratamentos para o período de incubação (Tabela 1). A porcentagem de eclosão dos ovos também não foi influenciada pela aplicação das suspensões fúngicas (Tabela 1).

Tabela 1. Período de incubação \pm EP (dias) e porcentagem de eclosão dos ovos de Chrysoperla externa (Neuroptera: Chrysopidae) tratada com diferentes suspensões do fungo Metarhizium anisopliae (Hypocreales: Clavicipitaceae)

\begin{tabular}{ccc}
\hline $\begin{array}{c}\text { Tratamentos } \\
\left(\text { Conídios } \mathbf{~ L}^{-1}\right)\end{array}$ & $\begin{array}{c}\text { Período de } \\
\text { incubação (dias) }\end{array}$ & Eclosão (\%) \\
\hline Testemunha & $3,56 \pm 0,14 \mathrm{a}$ & $100,00 \pm 0,00 \mathrm{a}$ \\
$10^{5}$ & $3,67 \pm 0,18 \mathrm{a}$ & $95,00 \pm 5,00 \mathrm{a}$ \\
$10^{6}$ & $3,63 \pm 0,24 \mathrm{a}$ & $95,00 \pm 5,00 \mathrm{a}$ \\
$10^{7}$ & $4,06 \pm 0,14 \mathrm{a}$ & $95,00 \pm 5,00 \mathrm{a}$ \\
$10^{8}$ & $4,00 \pm 0,11 \mathrm{a}$ & $75,00 \pm 9,57 \mathrm{a}$ \\
\hline F (tratamento) & $\mathbf{7 , 5 1 ^ { \text { ns } }}$ & $\mathbf{2 , 8 5 ^ { \text { ns } }}$ \\
\hline C.V. (\%) & $\mathbf{2 , 2 1}$ & $\mathbf{1 2 , 5 5}$ \\
\hline
\end{tabular}

Médias \pm erro-padrão(EP), seguidas por letras distintas na coluna diferem entre si pelo teste de Tukey $(\mathrm{P}<0,05)$. Dados originais; para análise foram transformados em $(\mathrm{x}+\mathrm{O}, 5)^{1 / 2}$

Em contradição, MagalHães et al. (1998) citam que os agentes entomopatogênicos quando aplicados sobre o predador, em contato com plantas contaminadas, ou ainda se alimentando de presas infectadas podem inviabilizar os ovos, o que não ocorreu com os resultados encontrados na presente pesquisa.

A porcentagem de eclosão encontrada para as suspensões de $10^{5}, 10^{6}$ e $10^{7}$ conídios. $\mathrm{mL}^{-1}$ de suspensão de $M . a$ foi de $95,00 \%$, ficando próximo do identificado por PessoA et al. (2005), que verificaram eclosão superior a 96,00\% quando ovos de C. externa foram tratados com isolados de outro fungo entomopatogênicos, Beauveria bassiana (Balsamo-Crivelli)Vuillemin (IBCB 66) (Ascomycetes: Clavicipitaceae).

Verificam-se diferenças significativas entre as várias doses de fungo utilizadas na duração dos instares e fase larval e, suas respectivas viabilidades (Tabela 2 ).

Larvas de primeiro instar de C. externa foram menos suscetíveis quando submetidas à dose de $10^{6}$ conídios.mL ${ }^{-1}$ de suspensão de M.a (2,35 dias) em comparação às larvas tratadas com as concentrações de $10^{5}$ e $10^{8}$ conídios.mL $\mathrm{m}^{-1}$ de suspensão de $M . a(2,0$ dias respectivamente). Nas concentrações de $10^{5}$ e $10^{8}$ conídios. $\mathrm{mL}^{-1}$ de $M . a$, a duração (dias) do estádio larval foi menor, diferindo significativamente da dose de $10^{6}$ conídios. $\mathrm{mL}^{-1}$ de suspensão de M.a. A viabilidade no primeiro instar variou de 95 a 100\%, não ocorrendo diferenças significativas para esse parâmetro.

No segundo instar, as larvas foram menos suscetíveis quando aplicado à dose de $10^{6}$ conídios.mL $\mathrm{LL}^{-1}$ de suspensão de M.a. (1,93 dias), diferindo significativamente da dose de $10^{8}$ conídios. $\mathrm{mL}^{-1} \mathrm{de}$ suspensão de M.a. (1,33 dias). Destaca-se que a troca de estádio nesta concentração conídios.mL $\mathrm{mL}^{-1}$ de suspensão de $M$.a. foi mais rápida. A viabilidade no segundo instar larval foi de $100 \%$, independentemente do tratamento, enquanto a viabilidade do terceiro instar variou de 95 a 100\%.

Com relação ao terceiro instar, a dose menos prejudicial às larvas foi a de $10^{7}$ conídios.mL ${ }^{-1}$ de suspensão de M.a., na qual o período de duração foi de dois dias, diferindo significativamente da dose de10 ${ }^{8}$ conídios.mL $L^{-1}$ de suspensão. PEssoA et al. (2005) observaram diminuição desse período quando as larvas foram tratadas com as suspensões de $1 \times 10^{7}$ e $1 \times 10^{8}$ conídios.mL $L^{-1}$ de suspensões de B. bassiana.

A redução na duração na fase larval (dias) implica em menor consumo do predador C. externa, considerando que é grande o consumo dos indivíduos na fase larval do mesmo, onde o terceiro estágio larval possui a maior capacidade predatória (BоRTOLI et al. 2006).

De modo geral, a fase larval foi menos prejudicada quando aplicada a dose de tratamento $10^{6}$ conídios.mL $L^{-1}$ de suspensão de M.a., e mais prejudicada quando utilizada a dose de10 ${ }^{8}$ conídios. $\mathrm{mL}^{-1}$ de suspensão de M.a., diferindo significativamente entre si. A viabilidade da fase larval variou de 95,00 a 100\%. Generoso (2002), avaliando os fungos B. bassiana (CG 149) (Ascomycetes: Clavicipitaceae) e Paecilomyces fumosoroseus Banir (JAB 12) (Deuteromycotina: Hyphomycetes) constatou seletividade em relação às larvas de primeiro e terceiro instar de $C$. externa. O mesmo não foi observado por Ventura et al. (1996), que relataram em condições de laboratório o efeito negativo do fungo $M$. anisopliae sobre o terceiro instar de Chrysoperla kolthofi (Navás) (Neuroptera: Chrysopidae), com concentrações que variaram de $1,5 \times 10^{4}$ a $1,5 \times 10^{12}$ conídios.mL ${ }^{-1}$. A partir da concentração de $1,5 \times 10^{8}$ conídios.mL $\mathrm{mL}^{-1}$,as larvas se mostraram suscetíveis ao fungo, atingindo $100 \%$ de mortalidade na maior concentração de $1,5 \times 10^{12}$ conídios.mL $\mathrm{mL}^{-1}$.

Especula-se que o fato de a testemunha na presente pesquisa ter apresentado médias intermediárias às dosagens testadas em todos os ínstares do predador seja por ter-se utilizado uma suspensão de $100 \mathrm{~mL}^{-1}$ de água deionizada sobre as larvas, o que possivelmente ocasionou um estresse, reduzindo assim o seu ciclo biológico. MesQuita et al. (1999) não encontraram diferença utilizando $0,2 \mathrm{~mL}^{-1}$ de água destilada esterilizada sobre disco de

Tabela 2. Duração \pm EP (dias) e viabilidade (\%) das fases de desenvolvimento de Chrysoperla externa (Neuroptera: Chrysopidae) tratadas com diferentes suspensões de Metarhizium anisopliae (Hypocreales: Clavicipitaceae)

\begin{tabular}{|c|c|c|c|c|c|c|c|c|}
\hline \multirow{2}{*}{$\begin{array}{c}\text { Tratamentos } \\
\text { (Conídios } \\
\text { mL }^{-1} \text { ) }\end{array}$} & \multicolumn{2}{|c|}{ Primeiro instar } & \multicolumn{2}{|c|}{ Segundo instar } & \multicolumn{2}{|c|}{ Terceiro instar } & \multicolumn{2}{|c|}{ Fase larval } \\
\hline & $\begin{array}{l}\text { Duração } \\
\text { (dias) }\end{array}$ & $\begin{array}{c}\text { Viabilidade } \\
\text { (\%) }\end{array}$ & $\begin{array}{l}\text { Duração } \\
\text { (dias) }\end{array}$ & $\begin{array}{c}\text { Viabilidade } \\
\text { (\%) }\end{array}$ & $\begin{array}{l}\text { Duração } \\
\text { (dias) }\end{array}$ & $\begin{array}{c}\text { Viabilidade } \\
\text { (\%) }\end{array}$ & $\begin{array}{l}\text { Duração } \\
\text { (dias) }\end{array}$ & $\begin{array}{c}\text { Viabilidade } \\
\text { (\%) }\end{array}$ \\
\hline Testemunha & $2,21 \pm 0,11 a b$ & $95,00 \pm 5,00 \mathrm{a}$ & $1,71 \pm 0,13 a b$ & $100,00 \pm 0,00 \mathrm{a}$ & $1,92 \pm 0,18 a b$ & $100,00 \pm 0,00 \mathrm{a}$ & $5,85 \pm 0,19 a b$ & $95,00 \pm 5,00 \mathrm{a}$ \\
\hline $10^{5}$ & $2,00 \pm 0,00 \mathrm{~b}$ & $100,00 \pm 0,00 \mathrm{a}$ & $1,60 \pm 0,13 a b$ & $100,00 \pm 0,00 \mathrm{a}$ & $1,47 \pm 0,13 a b$ & $100,00 \pm 0,00 \mathrm{a}$ & $5,07 \pm 0,23 b c$ & $100,00 \pm 0,00 \mathrm{a}$ \\
\hline $10^{6}$ & $2,35 \pm 0,13 a$ & $95,00 \pm 5,00 \mathrm{a}$ & $1,93 \pm 0,07 \mathrm{a}$ & $100,00 \pm 0,00 \mathrm{a}$ & $1,86 \pm 0,14 a b$ & $100,00 \pm 0,00 \mathrm{a}$ & $6,14 \pm 0,25 \mathrm{a}$ & $95,00 \pm 5,00$ a \\
\hline $10^{7}$ & $2,20 \pm 0,10 a b$ & $100,00 \pm 0,00 \mathrm{a}$ & $1,73 \pm 0,15 \mathrm{ab}$ & $100,00 \pm 0,00 \mathrm{a}$ & $2,00 \pm 0,29 a$ & $100,00 \pm 0,00 \mathrm{a}$ & $5,93 \pm 0,32 \mathrm{ab}$ & $\begin{array}{c}100,00 \pm \\
0,00 \mathrm{a}\end{array}$ \\
\hline $10^{8}$ & $2,00 \pm 0,00 \mathrm{~b}$ & $100,00 \pm 0,00 \mathrm{a}$ & $1,33 \pm 0,13 b$ & $100,00 \pm 0,00 \mathrm{a}$ & $1,21 \pm 0,11 \mathrm{~b}$ & $95,00 \pm 5,00 \mathrm{a}$ & $4,50 \pm 0,23 \mathrm{c}$ & $95,00 \pm 5,00$ a \\
\hline $\begin{array}{c}\mathrm{F} \\
\text { (tratamento) }\end{array}$ & $2,92^{*}$ & $0,75^{\text {ns }}$ & $3,08^{*}$ & $\mathbf{0}, \mathbf{O O}^{\text {ns }}$ & $3,44^{*}$ & $1, \mathbf{O O}^{\text {ns }}$ & $6,92^{* *}$ & $\mathbf{0 , 5 0 ^ { \text { ns } }}$ \\
\hline C.V. (\%) & 6,10 & 6,45 & 11,56 & $\mathbf{0 , 0 0}$ & 15,32 & 4,52 & 8,35 & 7,99 \\
\hline
\end{tabular}

Médias \pm erro-padrão (EP), seguidas por letras distintas na coluna diferem entre si pelo teste de Tukey (P<0,05). Dados originais. Sem transformação para análise. 
T papel.

Verificou-se que larvas do segundo instar apresentaram menor peso médio quando aplicado na dosagem de $10^{5}$ conídios. $\mathrm{mL}^{-1}$ de suspensão de M.a. (2,2 mg) diferindo significativamente da dosagem $10^{8}$ conídios.mL ${ }^{-1}$ de suspensão de M.a., com maior valor (2,6 mg) (Tabela 3). Observa-se que a dosagem $10^{8}$ conídios. $\mathrm{mL}^{-1}$ de suspensão de M.a. foi à mesma que acelerou a troca dos ínstares na fase larval em relação às demais, o que possivelmente pode ter influenciado as larvas a se alimentarem mais adquirindo maior peso.

Com relação ao peso médio de larvas de terceiro ínstar, não ocorreram diferenças significativas entre os tratamentos, porém, os valores estiveram entre 6,12 a $7,50 \mathrm{mg}$.

Não houve diferença significativa entre as doses de $M$. anisopliae aplicadas sobre a duração da fase pupal de C. externa (Tabela 4). Provavelmente, nesse período o crisopídeo não sofre interferência da aplicação do fungo $M$. anisopliae, o que possivelmente pode ser explicado pela fase pupal estar envolvida por fios de seda endurecidos em secreções produzidas pelos tubos de Malpighi (Trivellato et al. 2012). Deste modo, nessa pesquisa, o fungo não influenciou a fase de pupa por não entrar em contato com o tegumento para a posterior germinação. Provavelmente, a seda proporciona uma barreira física e mecânica que impede a penetração do fungo, corroborando os resultados de Silva et al. (2006), que não observaram ação de produtos fitossanitários sobre a pupa e adultos de C. externa.

A porcentagem de emergência dos adultos variou de 95\% para as maiores doses testadas e $100 \%$ para as demais. Mesmo sendo elevado o índice de emergência, notaram-se algumas deformidades nos adultos, sendo que alguns emergiram com as asas deformadas. No entanto, esse fato ocorreu sob todas as doses de $M$. anisopliae e também na testemunha, e, portanto, é decorrente de ordem genética e não do efeito das soluções aplicadas. Todavia, para as doses $\mathrm{de} 1 \mathrm{O}^{7}$ e $10^{8}$ conídios. $\mathrm{mL}^{-1}$ de suspensão de M.a. alguns indivíduos emergiram com essa deformação e posteriormente morreram, devido à ação do fungo.

A longevidade de C. externa tratada com diferentes suspensões do fungo $M$. anisopliae não foi verificado diferenças entre os tratamentos avaliados, com as médias variando de 17,33 dias para a testemunha, e 21,86 dias para indivíduos tratados com $10^{6}$ (Tabela 5).

Com relação à capacidade de oviposição, observou-se diferença significativa entre as médias dos tratamentos. Para a dose de $10^{5}$ conídios. $\mathrm{mL}^{-1}$ de suspensão de M.a. obteve-se 14,38 ovos, que diferiu significativamente da dose de $10^{6}$ conídios. $\mathrm{mL}^{-1}$ de suspensão de M.a. com 6,97 ovos, e da dose de10 ${ }^{7}$ conídios.mL $\mathrm{mL}^{-1}$ de suspensão de M.a. com 5,93 ovos. Nota-se que o fungo não interferiu na longevidade dos adultos, porém, influenciou na oviposição, destacando-se as doses de $10^{6}, 10^{7}$ e $10^{8}$ conídios. $\mathrm{mL}^{-1}$ de suspensão de M.a. como as prejudiciais. Esse tipo de teste é importante, pois proporcionam uma estimativa do que irá ocorrer com os indivíduos nas gerações seguintes, quando esses anteriormente tiveram contato com determinadas substâncias.

Tabela 3. Biomassa \pm EP (mg) de larvas de Chrysoperla externa (Neuroptera: Chrysopidae) tratadas com diferentes suspensões de Metarhizium anisopliae (Hypocreales: Clavicipitaceae).

\begin{tabular}{ccc}
\hline \multirow{2}{*}{ Tratamentos (Conídios $\mathbf{~ m L}^{-1}$ ) } & \multicolumn{2}{c}{ Biomassa \pm EP (mg) } \\
\cline { 2 - 3 } & $\mathbf{2}^{\mathbf{0} \text { instar }}$ & $\mathbf{3}^{\mathbf{0}}$ instar \\
\hline Testemunha & $2,40 \pm 0,12 \mathrm{ab}$ & $6,12 \pm 0,44 \mathrm{a}$ \\
$10^{5}$ & $2,20 \pm 0,09 \mathrm{~b}$ & $7,36 \pm 0,39 \mathrm{a}$ \\
$10^{6}$ & $2,40 \pm 0,08 \mathrm{ab}$ & $7,78 \pm 0,31 \mathrm{a}$ \\
$10^{7}$ & $2,30 \pm 0,06 \mathrm{ab}$ & $7,12 \pm 0,31 \mathrm{a}$ \\
\hline $10^{8}$ & $2,60 \pm 0,08 \mathrm{a}$ & $7,50 \pm 0,59 \mathrm{a}$ \\
\hline F (tratamento) & $\mathbf{2 , 8 0 ^ { * }}$ & $\mathbf{1 , 6 7 ^ { \mathbf { n s } }}$ \\
\hline C.V. (\%) & $\mathbf{1 1 , 4 7}$ & $\mathbf{9 , 0 3}$ \\
\hline
\end{tabular}

Médias \pm erro-padrão (EP), seguidas por letras distintas na coluna diferem entre si pelo teste de Tukey $(\mathrm{P}<0,05)$. Dados originais; para análise foram transformados em $(\mathrm{x}+\mathrm{O}, 5)^{1 / 2}$.

Tabela 4. Duração da fase pupal \pm EP (dias) e porcentagem de emergência (\%) de Chrysoperla externa (Neuroptera: Chrysopidae) tratada com diferentes suspensões de Metarhizium anisopliae (Hypocreales: Clavicipitaceae).

\begin{tabular}{|c|c|c|}
\hline Tratamento (Conídios $\mathbf{m L}^{-1}$ ) & Duração (dias) & Emergência (\%) \\
\hline Testemunha & $10,06 \pm 0,18 a$ & $100,00 \pm 0,00 \mathrm{a}$ \\
\hline $10^{5}$ & $10,06 \pm 0,20 \mathrm{a}$ & $100,00 \pm 0,00 \mathrm{a}$ \\
\hline $10^{6}$ & $10,40 \pm 0,13 a$ & $100,00 \pm 0,00 \mathrm{a}$ \\
\hline $10^{7}$ & $9,93 \pm 0,20 \mathrm{a}$ & $95,00 \pm 5,00 \mathrm{a}$ \\
\hline $10^{8}$ & $10,46 \pm 0,21 \mathrm{a}$ & $95,00 \pm 5,00 \mathrm{a}$ \\
\hline F (tratamento) & $1,49^{\text {ns }}$ & $0,75^{\text {ns }}$ \\
\hline C.V. (\%) & 7,25 & 6,45 \\
\hline
\end{tabular}

Médias \pm erro-padrão (EP), seguidas por letras distintas na coluna, diferem entre si pelo teste de Tukey $(\mathrm{P}<0,05)$. Dados originais; sem transformação para análise.

Tabela 5. Longevidade (dias) e capacidade de oviposição média \pm EP de Chrysoperla externa (Neuroptera: Chrysopidae) tratada com suspensões de Metarhizium anisopliae (Hypocreales: Clavicipitaceae).

\section{Tratamentos(Conídios $\mathrm{mL}^{-1}$ )} Testemunha

$10^{5}$

$10^{6}$

$10^{7}$

$10^{8}$

F (tratamento)

C.V. (\%)
Longevidade (dias)

$17,33 \pm 2,8 \mathrm{a}$

$21,20 \pm 3,4 \mathrm{a}$

$21,86 \pm 3,6$ a

$17,60 \pm 4,9 a$

$21,80 \pm 6,3$ a

$0,27^{\mathrm{ns}}$

Capacidade de oviposição

$10,93 \pm 1,9 \mathrm{a}$

$14,38 \pm 2,1 \mathrm{a}$

$6,97 \pm 1,5 \mathrm{~b}$

$5,93 \pm 0,8 b$

$8,72 \pm 1,3 \mathrm{ab}$

$4,65^{* * *}$

Médias \pm erro-padrão (EP), seguidas por letras distintas na coluna diferem entre si pelo teste de Tukey $(\mathrm{P}<0,05)$. Dados originais; para a análise foram transformados em $(\mathrm{x}+\mathrm{O}, 5)^{1 / 2}$. 
Aspectos biológicos de $C$. externa alimentada com B. tabaci biótipo B criada sobre folhas de algodoeiro tratado com o fungo. A duração dos estágios larvais de $C$. externa alimentada com $B$. tabaci biótipo $\mathrm{B}$, anteriormente tratadas com o fungo $M$. anisopliae encontra-se na Tabela 6 . Não se observou diferenças significativas entre os tratamentos para duração dos ínstares de C. externa. A duração variou de 3,81 a 4,00 dias para o primeiro ínstar, 3,00 a 3,35 dias para o segundo instar, no terceiro instar 7,21 a 8,90 dias e de 14,50 a 15,75 para a fase larval completa.

Esses resultados não são condizentes com os relatados por Magalhães et al. (1998), que indicam que os fungos entomopatogênicos podem agir de forma prejudicial sobre os predadores, alterando seu desenvolvimento biológico.

Como observado no segundo ínstar da mosca-branca, em geral, obteve-se uma menor duração do ínstar em relação ao primeiro e terceiro ínstares, o que também foi observado por MAIA et al. (2000), utilizando larvas de C. externa. Independentemente do tipo de alimento ingerido pelas larvas, a duração do segundo ínstar variou de 4,3 a 4,8 dias, sendo assim menor do que a dos demais ínstares, e provavelmente é uma característica biológica desse predador.

A viabilidade larval de $C$. externa no tratamento testemunha se manteve em 100\% no primeiro e segundo ínstares, não ocorrendo o mesmo para o tratamento com de maior dose $(85 \%$ de viabilidade larval para os mesmos ínstares). Com relação à fase larval, a dose de $10^{8}$ conídios. $\mathrm{mL}^{-1}$ de suspensão de M.a. apresentou $24 \%$ de viabilidade.

Verificou-se que as fases de pré-pupa e pupa não foram afetadas significativamente entre os tratamentos testados, variando de 4,10 a 4,25 dias para a duração do período de pré-pupa e de 5,25 a 6,00 dias para o período de pupa (Tabela 7). Porém, observa-se uma diminuição da viabilidade de pré-pupa com o tratamento com a dose de $10^{5}$ conídios. $\mathrm{mL}^{-1}$ de suspensão de M.a. (65\%),diferindo significativamente da testemunha (95\%). As larvas de terceiro ínstar não transformaram adequadamente em pupa, pois não construíram o casulo na dose de $10^{5}$ conídios. $\mathrm{mL}^{-1}$ de suspensão e o mesmo ocorreram para a dose de $10^{8}$ conídios. $\mathrm{mL}^{-1}$ de suspensão de M.a.

Para as pupas tratadas com a dose de $10^{8}$ conídios. $\mathrm{mL}^{-1}$ de suspensão de M.a. a emergência dos adultos foi de $75 \%$, comum à redução de $21 \%$ em relação à testemunha, enquanto pupas tratadas com a dose de $10^{5}$ conídios. $\mathrm{mL}^{-1}$ de suspensão obteve 90\% de emergência de adultos. Ambas as doses apresentaram essa diminuição devido à mortalidade da pupa, que apresentou coloração escura e seca.

Entre as doses testadas nesse trabalho, $10^{5}$ conídios. $\mathrm{mL}^{-1}$ de suspensão com o fungo $M$. anisopliae foi menos prejudicial quando aplicada diretamente no predador, e as doses de $10^{5} \mathrm{e}$ $10^{8}$ conídios. $\mathrm{mL}^{-1}$ de suspensão não interferiram nos aspectos biológicos de $C$. externa quando oferecidas ninfas de $B$. tabaci biótipo B após 24 horas da aplicação. De maneira geral, é possível realizar o tratamento com suspensões do fungo $M$. anisopliae, e após as 24 horas, realizar uma liberação massal de C. externa, proporcionando uma associação harmoniosa entre esses agentes biológicos para o manejo de $B$. tabaci biótipo $\mathrm{B}$ na cultura do algodoeiro,

Verificou-se no presente estudo a existência de compatibilidade das doses de $10^{5}$ e $10^{8}$ conídios. $\mathrm{mL}^{-1}$ de suspensão do fungo $M$. anisopliae sobre os aspectos biológicos de C. externa, sendo possível realizar a liberação deste predador 24 horas após a aplicação das suspensões deste entomopatôgeno no algodoeiro.

Tabela 6. Duração \pm EP (dias) e viabilidade (\%) das fases de desenvolvimento de Chrysoperla externa (Neuroptera: Chrysopidae) alimentada com B. tabaci biótipo B oriunda de folhas de algodoeiro, tratadas com diferentes suspensões de Metarhizium anisopliae (Hypocreales: Clavicipitaceae).

\begin{tabular}{|c|c|c|c|c|c|c|c|c|}
\hline \multirow{2}{*}{$\begin{array}{c}\text { Tratamento } \\
\text { (Conídios/ } \\
\text { mL) }\end{array}$} & \multicolumn{2}{|c|}{ Primeiro instar } & \multicolumn{2}{|c|}{ Segundo instar } & \multicolumn{2}{|c|}{ Terceiro instar } & \multicolumn{2}{|c|}{ Fase larval } \\
\hline & $\begin{array}{c}\text { Duração } \\
\text { (dias) }\end{array}$ & $\begin{array}{c}\text { Viabilidade } \\
\text { (\%) }\end{array}$ & $\begin{array}{l}\text { Duração } \\
\text { (dias) }\end{array}$ & $\begin{array}{c}\text { Viabilidade } \\
\text { (\%) }\end{array}$ & $\begin{array}{c}\text { Duração } \\
\text { (dias) }\end{array}$ & $\begin{array}{c}\text { Viabilidade } \\
\text { (\%) }\end{array}$ & $\begin{array}{l}\text { Duração } \\
\text { (dias) }\end{array}$ & $\begin{array}{c}\text { Viabilidade } \\
\text { (\%) }\end{array}$ \\
\hline Testemunha & $3,83 \pm 0,14 a$ & $100,00 \pm 0,00 \mathrm{a}$ & $3,05 \pm 0,20 \mathrm{a}$ & $100,00 \pm 0,00 \mathrm{a}$ & $8,66 \pm 0,51 \mathrm{a}$ & $85,00 \pm 9,57 \mathrm{a}$ & $15,75 \pm 0,59 a$ & $85,00 \pm 9,57 \mathrm{a}$ \\
\hline $10^{5}$ & $4,00 \pm 0,17 a$ & $85,00 \pm 9,57 \mathrm{a}$ & $3,35 \pm 0,26 a$ & $85,00 \pm 5,00$ a & $7,21 \pm 0,49 a$ & $100,00 \pm 0,00 \mathrm{a}$ & $14,50 \pm 0,71 \mathrm{a}$ & $70,00 \pm 12,91 \mathrm{a}$ \\
\hline $10^{8}$ & $3,81 \pm 0,10 \mathrm{a}$ & $85,00 \pm 5,00$ a & $3,00 \pm 0,16 \mathrm{a}$ & $85,00 \pm 9,57 \mathrm{a}$ & $8,90 \pm 0,66 \mathrm{a}$ & $95,00 \pm 5$, ooa & $15,73 \pm 0,73 \mathrm{a}$ & $65,00 \pm 12,58 \mathrm{a}$ \\
\hline $\begin{array}{c}\mathrm{F} \\
\text { (tratamento) }\end{array}$ & $\mathbf{0 , 5 1 ^ { \text { ns } }}$ & $1,93^{\text {ns }}$ & $0,75^{\text {ns }}$ & $1,93^{\text {ns }}$ & $\mathbf{2}, \mathbf{8 9} 9^{\mathrm{ns}}$ & $\mathbf{1}, \mathbf{5 0}^{\mathrm{ns}}$ & $1,16^{\mathrm{ns}}$ & $\mathbf{0}, 7^{\text {ns }}$ \\
\hline C.V. (\%) & 15,30 & 13,86 & 26,42 & 13,86 & 23,6 & 13,36 & 15,73 & 32,14 \\
\hline
\end{tabular}

Médias \pm erro-padrão (EP), seguidas por letras distintas na coluna diferem entre si pelo teste de Tukey (P<0,05). Dados originais. Sem transformação para análise.

Tabela 7. Duração \pm EP (dias) e viabilidade (\%) da pré-pupa e pupa de Chrysoperla externa (Neuroptera: Chrysopidae) alimentada com $B$. tabaci biótipo B oriunda de folhas de algodoeiro tratadas com suspensões de Metarhizium anisopliae (Hypocreales: Clavicipitaceae).

\begin{tabular}{|c|c|c|c|c|}
\hline \multirow{2}{*}{$\begin{array}{c}\text { Tratamento } \\
\text { (Conídios } \mathrm{mL}^{-1} \text { ) }\end{array}$} & \multicolumn{2}{|c|}{ Pré-Pupa } & \multicolumn{2}{|c|}{ Pupa } \\
\hline & Duração (dias) & Viabilidade (\%) & Duração (dias) & Emergência (\%) \\
\hline Testemunha & $4,10 \pm 0,23 a$ & $95,00 \pm 5,00 \mathrm{a}$ & $6,00 \pm 0,42 \mathrm{a}$ & $95,00 \pm 5,00 \mathrm{a}$ \\
\hline $10^{5}$ & $4,25 \pm 0,51 \mathrm{a}$ & $65,00 \pm 9,57 b$ & $5,66 \pm 0,42 \mathrm{a}$ & $90,00 \pm 5,77 \mathrm{a}$ \\
\hline $10^{8}$ & $4,11 \pm 0,66 \mathrm{a}$ & $90,00 \pm 5,77 \mathrm{ab}$ & $5,25 \pm 0,47 a$ & $75,00 \pm 12,58 \mathrm{a}$ \\
\hline F (tratamento) & $0,08^{\text {ns }}$ & $5,17^{*}$ & $0,59^{\text {ns }}$ & $1,5^{0^{n s}}$ \\
\hline C.V. (\%) & 20,46 & 16,97 & 20,69 & 19,61 \\
\hline
\end{tabular}

Médias \pm erro-padrão (EP), seguidas por letras distintas na coluna diferem entre si pelo teste de Tukey (P<0,05). Dados originais; Sem transformação para análise.

\section{REFERÊNCIAS}

Alves, S.B.; R.B. Lopes, 2008. Controle microbiano de pragas na América Latina: avanços e desafios. Piracicaba: FEALQ, 414 p.

Azevedo, F.R. de, J.A. Guimarães, R. Braga Sobrinho, M.A.A. Lima, 2005. Eficiência de produtos naturais para o controle de Bemisia tabaci biótipo b (Hemíptera: Aleyrodidae) em meloeiro. Arquivos Instituto Biológico, 72:73-79.

Auad, A.M., L.C. Toscano, A.L. Boiça Júnior \& S. Freitas, 2001. Aspectos biológicos dos estádios imaturos de Chrysoperla externa (Hagen) e Ceraeochrysa cincta (Schneider) (Neuroptera: Chrysopidae) alimentados com ovos e ninfas de Bemisia tabaci (Gennadius) biótipo B (Hemiptera: 
Aleyrodidae). Neotropical Entomology,30: 429-432.

Auad, A.M., C.F. Carvalho, B. Souza, R. Trevizani \& C.M.F.R. Magalhães, 2005. Desenvolvimento das fases imaturas, aspectos reprodutivos epotenciais de predação de Chrysoperla externa alimentada com ninfas de Bemisia tabaci biótipo B em tomateiro. Acta Scientiarum Agronomy, 27: 327-334.

Barbosa, L.R., C.F. Carvalho, B. Souza \& A.M. Auad, 2008. Eficiência de Chrysoperla externa (Hagen, 1861) (Neuroptera Chrysopidae) no controle de Myzus persicae (Sulzer, 1776) (Hemiptera: Aphididae) em pimentão (Capsicumannum L.). Ciência e Agrotecnologia, 32: 1113-1119.

Berry, S.D., V.N. London, C. Rey, D. Rogan, C.M. Fauquet \& J.K. Brown, 2004. Molecular evidence for five distinct Bemisia tabaci (Homoptera: Aleyrodidae) geographic haplotypes associated with cassava plants in Sub Saharan Africa. Annals ofthe Entomological Society of America, 97: 852-859.

Bezerra, C.E.S, C.H.F. Nogueira, K.D.S. Sombra, A.C.F. Demartelaere \& E.L. Araújo, 2009.Crisopídeos (Neuroptera: Chrysopidae): aspectos biológicos, potencial de utilização e perspectivas futuras. Revista Caatinga, 22: 1-5.

Bortoli, S.A., A.C. Caetano, A.T. Murata, S.A. Oliveira, A.L.F. Braga \& R.B. Ferreira, 2006. Potencial de Chrysoperla externa (Hagen) (Neuroptera: Chrysopidae) em diferentes presas. Biologia e Ciências da Terra, 6: 145-153.

Bueno, A. de F., D.R. Sosa-Gómez, B.S. Corrêa-Ferreira, F. Moscardi \& R.C.O.F Bueno, 2012. Inimigos naturais das pragas da soja. In: (Ed.). Soja: manejo integrado de insetos e outros artrópodes-praga, 493-629p.

Butler Junior, G.D., F.D. Wilson \& G. Fishler, 1991.Cotton leaf trichomes and populations of Empoasca lybica and Bemisia tabaci. Crop Protection, 10: 461-464.

Carvalho, C.F. \& B. Souza, 2009. Métodos de criação e produção de crisopídeos, p.77-115. In: Bueno, V.H.P. (Ed.). Controle biológico de pragas: produção massal e controle de qualidade. 2 ed., UFLA, 115p.

França, Í.W.B., E.J. Marques, J.B. Torres \& J.V. Oliveira, 2006. Efeitos de Metarhizium anisopliae (Metsch.) Sorok. E Beauveria bassiana (Bals.) Vuill. sobre o Percevejo Predador Podisus nigrispinus (Dallas) (Hemiptera: Pentatomidae). Neotropical Entomology, 35: 349-356.

Freitas, S., 2003. Chrysoperla steinmanni, 1964 (Neuroptera: Chrysopidae): descrição de uma nova espécie do Brasil. Revista Brasileira de Entomologia, 47: 385-387.

Gassen,M.H.,2006.Patogenicidadedefungos entomopatogênicos para o psilídeo da goiabeira Triozoida sp. (Hemíptera: Psyllidae) e compatibilidade de agrotóxicos utilizados na cultura da goiaba sobre estes agentes de controle biológico. 110 p.Dissertação (Mestrado em Agronomia) - Faculdade de Ciências Agronômicas da UNESP, Botucatu, SP.

Generoso, A.R., 2002. Compatibilidade de Beauveria bassiana e Paecilomyces fumosoroseus com Chrysoperla externa (Neuroptera: Chrysopidae) e metodologia para avaliação da seletividade. 63p. Dissertação (Mestrado em Agronomia) Universidade Estadual Paulista, Jaboticabal-SP.

Gravena, S. 2000. Os fungos no controle de insetos. Revista Cultivar, 15: 41-43.

Loureiro, E.S., A. Batista Filho, J.E.M. Almeida, J.M. Mendes \& L.G.A. Pessoa, 2012. Eficiência de isolados de Metarhizium anisopliae (Metsch.) Sorok. no controle da cigarrinha-daraiz da cana-de-açúcar, Mahanarva fimbriolata (Stal, 1854) (Hemiptera: Cercopidae), em condições de campo. Arquivo do Instituto Biológico,79: 47-53.

Maia, W.J.M.S., C.F. Carvalho\& B. Souza, 2000. Exigências térmicas de Chrysoperla externa (Hagen, 1861) (Neuroptera: Chrysopidae) alimentada com Schizaphis graminum (Rondani, 1852) (Hemiptera: Aphididae) em condições de laboratório. Ciência e Agrotecnologia, 24: 81-86.

Magalhães, B.P., R. Monnerat \& S.B. Alves, 1998. Interações entre entompatógenos, parasitóides e predadores, p.195-216. In: ALVES, S. B. (Ed.). Controle microbiano de insetos. 2nd ed, Piracicaba, Fealq. 1163p.
Mesquita, A.L.M., L.A. Lacey, C. Ceianu \& R. Dabire, 1999. Predatory and parasiticactivity of Aphelinusasychis (Hymenoptera: Aphelinidae) following exposureto the entomopathogenic fungus Paecilomyces fumosoroseus (Deuteromycotina: Hyphomycetes) under different humidity regimes. Anais da Sociedade Entomológica do Brasil, 28: 661-673.

Padulla, L.F.L., 2007. Estudos de fungos entomopatogênicos para o controle de ninfas do psilídeo Diaphorina citri Kuwayama (Hemiptera: Psyllidae). 91p. Dissertação (Mestrado em Ciências) - Escola Superior de Agricultura "Luiz de Queiroz", Universidade de São Paulo, Piracicaba, SP.

Pessoa, L.G.A., R.S. Cavalcanti, A. Moino Júnior \& B. Souza, 2005. Compatibilidade entre Beauveria bassianae o predador Chrysoperla externa em laboratório. Pesquisa Agropecuária Brasileira, 40: 617-619.

Pinto, A. S. \& Parra, J.R.P., 2002. Liberação de inimigos naturais. In: Parra, J.R.P., P. S. M. Botelho; B. S. Corrêa-ferreira \& J. M. S. Bento. (Eds.). Controle biológico no Brasil: parasitóides e predadores. MANOLE, $635 \mathrm{p}$.

Ramiro, Z.A. \& Faria, A.M., 2006. Levantamento de insetos predadores nos cultivares de algodão Bollgard DP9o e convencional Delta Pine Acala 90. Arquivos Instituto Biológico, 73:119-121.

Ribeiro, M.J., 1988. Biologia de Chrysoperla externa (Hagen, 1861) (Neuroptera: Chrysopidae) alimentada com diferentes dietas. 131 p. Dissertação (Mestrado em agronomia) - Escola Superior de Agricultura de Lavras, Lavras-MG.

Rohde, C., L.F.A. Alves, P.M.O.J. Neves, S.B. Alves, E.R.L.da. Silva \& J.E.M. Almeida, 2006. Seleção de isolados de Beauveria bassiana (Bals.)Vuill. e Metarhizium anisopliae (Metsch). Sorok. contra o cascudinho Alphitobius diaperinus (Panzer) (Coleoptera:Tenebrionidae). Neotropical Entomology, 35: 231-240.

Silva, C.G., B. Souza, A.M. Auad, J.P. Bonani, L.C. Torres, C. F. Carvalho \& C.C. Ecole, 2004. Desenvolvimento das fases imaturas de Chrysoperla externa alimentadas com ninfas de Bemisia tabaci criadas em três hospedeiros. Pesquisa Agropecuária Brasileira, 39: 1065-1070.

Silva. R.A., G.A. Carvalho, C F. Carvalho, P.R. Reis, B. Souza \& A.M.A.R. Pereira, 2006. Ação de produtos fitossanitários utilizados em cafeeiros sobre pupas e adultos de Chrysoperla externa (Hagen, 1861) (Neuroptera: Chrysopidae). Ciência Rural, 36: 8-14.

Souza, A.L.V., 2013. Métodos de liberação de Chrysoperla externa (Hagen, 1861) (Neuroptera: Chrysopidae) visando o controle de Macrosiphum euphorbiae (Thomas, 1878) (Hemiptera: Aphididae) em roseiras sob cultivo protegido. 67p. Dissertação (Mestrado em Entomologia), Universidade Federal de Lavras, MG.

Schlick-Souza, E.C., L.C. Toscano, G.D. Souza-Schlick, E. Adriano, W. I. Maruyama, A.J.A.A. Peres, 2011. Capacidade predatória de Chrysoperla externa sobre Bemisia tabaci biótipo B expostas ao fungo Metarhizium anisopliae. Scientia Agraria,12: 121-126.

Tamai, M.A., 2002. Controle de Tetranychus uticae Koch com fungos entomopatogênicos. 144p. Tese (Doutorado em Ciências) - Escola Superior de Agricultura "Luiz de Queiroz", Universidade de São Paulo, Piracicaba, SP.

Trivellato G.F., 2012. Aspectos biológicos, e suas implicações na qualidade da produção massal de Chrysoperla externa (Hagem 1861) (Neuroptera, Chrysopidae) Dissertação (Mestrado em Entomologia) Universidade de São Paulo, Escola Superior de Agricultura Luiz de Queiroz. 68 p.

Van Emden, H. \& R. Harrington, 2007. Aphids as crop pests. Wallingford, CABI Publishing. $752 \mathrm{p}$.

Ventura, M.A., C. Ribeiro, V. Garcia, M. Canard, H. Aspock \& M. W. Mansell, 1996. Susceptibility of third instar larvae of the green lacewing Chrysoperla kolthoffi (Navás) to the entomopathogenic fungus Metarhizium anisopliae (Metschnikoff) Sorokin var. anisopliae Tulloch in the 
laboratory (Insecta: Neuroptera: Chrysopidae). Pure and applied research in neuropterology. Proceedings of The Fifth International Symposium on Neuropterology, 1: 241-249, 1996.

\section{Como citar este artigo:}

Souza, E.C.S., L.C. Toscano, G.D.S. Schlick, A.J.A. Peres, P.M. Dias \& W.I. Maryama, 2015. Compatibilidade de Metarhizium anisopliae (Metschnikoff) Sorokin (Hypocreales: Clavicipitaceae) com Chrysoperla externa (Hagen) (Neuroptera: Chrysopidae). EntomoBrasilis, 8 (3): 189195.

Acessível em: doi:10.12741/ebrasilis.v8i3.468
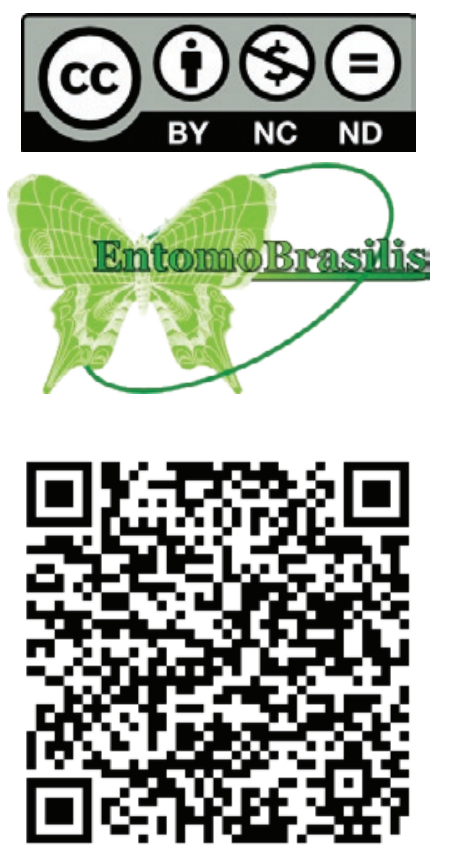